\title{
Lesiones del nervio cubital en el canal de Guyon: a propósito de 6 casos
}

\author{
S. M. Medina Macías ${ }^{(1)}$, J. S. OJeda Castellano ${ }^{(2)}$, M. Santana Vélez ${ }^{(2)}$, \\ R. Navarro García ${ }^{(2)}$ \\ (1) FREMAP-Gran Canaria (Patología del Miembro Superior) \\ (2) Servicio de C.O.T. del Hospital Universitario Insular de Gran Canaria
}

\author{
Correspondencia: \\ Dra. Sonia Ma Medina Macías \\ Dirección: Avda. de Canarias, 11 \\ 35110 Santa Lucía (Gran Canaria) \\ Teléfono: 928125324 \\ e-mail: sonia_medina@fremap.es
}

\begin{abstract}
La compresión del nervio cubital en el canal de Guyon es mucho menos frecuente que la compresión del nervio mediano en el canal carpiano o del nervio cubital en el codo, ocupando el tercer lugar dentro de los síndromes canaliculares del miembro superior, pero no por ello es rara. A diferencia de estos síndromes, es raro que aparezca de forma idiopática, siendo más frecuente que aparezca de forma secundaria a causas locales, por lo que, a parte del EMG, es más frecuente la utilización de otras pruebas complementarias (RM, TAC, Doppler) para completar el diagnóstico. También puede aparecer como consecuencia de microtraumatismos profesionales, $y$, dentro de las formas traumáticas, es muy rara la lesión aislada de la rama motora debido a un traumatismo penetrante.

Es fundamental conocer la anatomía de esta zona porque esto nos permitirá hacer un diagnóstico clínico relativamente fácil y precisar el lugar de la compresión, porque anatómicamente este canal se divide en tres zonas, pero además porque es preciso conocer las relaciones anatómicas de este espacio para evitar complicaciones o lesiones iatrogénicas del nervio cubital tras la cirugía del canal carpiano, que se pueden producir a través de la zona central de su pared interna que sólo está ocupada por tejido adiposo, pues el canal de Guyon no es un canal rígido sino de un conducto osteofibroso, tal como lo describió Guyon en 1861.
\end{abstract}

Palabras clave: canal de Guyon, secundario a causas locales, microtraumatismos profesionales, conducto osteofibroso
The compression of the ulnar nerve in the Guyon canal is much less common than compression of the median nerve in the carpal tunnel or ulnar nerve in the elbow, occupying the third place in the canaliculares syndromes of upper extremity, but that does not make it rare. Unlike these syndromes, it rarely appears in idiopathic form is most common to appear in a local secondary causes, so a part of EMG, it is more frequent use of other tests (MRI, CT, Doppler ) to complete the diagnosis. Can also occur as a result of microtraumatismos professional, and the ways in traumatic injury is rarely isolated from the motor injury due to penetrating trauma.

It is essential to know the anatomy of this area because this will allow us to make a clinical diagnosis and relatively easy to specify the location of the compression, because this canal is anatomically divided into three zones, but also because it is necessary to know the anatomical relationships of this space to avoid complications or iatrogenic injury of the ulnar nerve after carpal tunnel surgery, which may occur through the central area of its inner wall that is occupied only by adipose tissue, as the Guyon canal is not a rigid channel but a conduit osteofibroso, as described in Guyon 1861.

Key words: Guyon canal, Secondary to local causes, microtraumatismo profesional, conduit osteofibroso. 


\section{INTRODUCCIÓN}

$\mathrm{L}$ a compresión del nervio cubital en el canal de Guyon es mucho menos frecuente que la compresión del nervio mediano en el canal carpiano, pero no por ello es rara, pudiendo aparecer también como consecuencia de microtraumatismos profesionales o de forma traumática. El espacio fibroso descrito por Guyon en 1861 no es un canal rígido sino un conducto osteofibroso que contiene al paquete neurovascular cubital y sus divisiones ${ }^{1}$.

En su forma mixta o motriz pura, puede originar una amiotrofia grave y conducir a una parálisis total de los músculos intrínsecos de inervación cubital, por lo que el conocimiento de la anatomía de esta región permitirá hacer un diagnóstico clínico relativamente fácil y precisar cuál es el sitio de la compresión ${ }^{1}$.
Al llegar a la mano, el nervio cubital se sitúa por dentro de la arteria, tras desprenderse de la cara profunda del tendón del flexor carpi ulnaris, contorneando el borde externo de este tendón, y pasando enseguida por fuera del pisiforme y por delante del ligamento anular anterior. Está delimitado hacia atrás (suelo) por el ligamento anular anterior y por el ligamento pisiunciforme, hacia dentro (pared interna) por el pisiforme y hacia delante (techo) por un desdoblamiento palmar del ligamento anular anterior del carpo, una expansión del músculo flexor carpi ulnaris y el músculo palmaris brevis. Al salir de este conducto, es decir, a nivel del extremo inferior del pisiforme, y a veces en el mismo conducto, el nervio se divide en sus dos ramos terminales: ramas superficial y profunda ${ }^{2}$.

El canal de Guyon en la mano se divide en tres zonas: zona 1, que abarca desde la entrada

\section{Tabla I}

\begin{tabular}{|c|c|c|c|c|c|c|c|c|}
\hline & GÉNERO & EDAD & PROFESIÓN & D/I & $\begin{array}{c}\text { ZONA } \\
\text { LESIONAL }\end{array}$ & ETIOLOGÍA & $\begin{array}{c}\text { FORMA DE } \\
\text { PRSENTACIÓN }\end{array}$ & TRATAMIENTO \\
\hline $\begin{array}{c}\text { Caso } \\
1\end{array}$ & Varón & 25 & Mensajero & D & Zona 1 & $\begin{array}{l}\text { Traumática } \\
\text { (Cristal) }\end{array}$ & Aguda & $\begin{array}{c}\text { Apertura CG + Sutura } \\
\mathrm{T}-\mathrm{T} \text { de las ramas } \\
\text { motora y sensitiva }\end{array}$ \\
\hline $\begin{array}{c}\text { Caso } \\
2\end{array}$ & Varón & 34 & Agricultor & I & Zona 2 & $\begin{array}{l}\text { Traumática } \\
\text { (Objeto } \\
\text { penetrante) }\end{array}$ & Aguda & $\begin{array}{c}\text { Apertura CG + Sutura } \\
\text { de lesión parcial de la } \\
\text { rama motora }\end{array}$ \\
\hline $\begin{array}{c}\text { Caso } \\
3\end{array}$ & Varón & 38 & $\begin{array}{c}\text { Peón de la } \\
\text { Construcción }\end{array}$ & I & Zona 1 & $\begin{array}{c}\text { Traumática } \\
\text { (Fractura } \\
\text { EDR) }\end{array}$ & Subaguda & Conservador \\
\hline $\begin{array}{c}\text { Caso } \\
4\end{array}$ & Mujer & 31 & Panadera & D & Zona 1 & $\begin{array}{l}\text { latrogénica } \\
\text { (Liberación } \\
\text { CC) }\end{array}$ & Crónica & $\begin{array}{l}\text { Apertura CG + } \\
\text { Resección neuroma } \\
\text { y Sutura T-T de las } \\
\text { ramas motora y } \\
\text { sensitiva }\end{array}$ \\
\hline $\begin{array}{c}\text { Caso } \\
5\end{array}$ & Varón & 49 & Cocinero & D & Zona 2 & $\begin{array}{c}\text { Fractura } \\
\text { apófisis } \\
\text { unciforme del } \\
\text { ganchoso }\end{array}$ & Crónica & $\begin{array}{c}\text { Apertura CG + } \\
\text { Apertura del CC } \\
\text { - Sinovectomía de } \\
\text { flexores y tenodesis } \\
\text { del FDP del V al IV }\end{array}$ \\
\hline $\begin{array}{c}\text { Caso } \\
6\end{array}$ & Varón & 20 & Tapicero & I & Zona 2 & $\begin{array}{c}\text { Artrosis } \\
\text { del Carpo } \\
\text { (Seudoartrosis } \\
\text { de escafoides) }\end{array}$ & Crónica & Apertura del CG \\
\hline
\end{tabular}




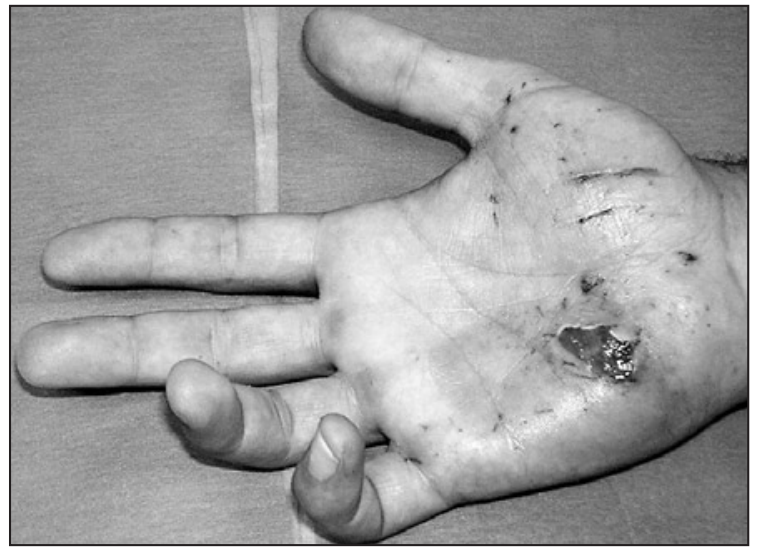

Figura1: Caso 1. Traumatismo penetrante (cristal). Garra cubital.

al canal de Guyon hasta la bifurcación del nervio cubital en sus ramas superficial y profunda (salida del canal); zona 2, que sólo contiene la rama profunda o motora que rodea la apófisis unciforme del hueso ganchoso por su lado interno, tras haber dado la rama destinada al músculo abductor digiti minimi, y pasa luego por debajo del arco fibroso de los hipotenares, e inerva además al flexor digiti minimi brevis y opponens digiti minimi, al adductor pollicis y al fascículo profundo del flexor pollicis brevis, a los Mn lumbricalis del $4^{\circ}$ y $5^{\circ}$ dedo y a los $\mathrm{Mn}$ interossei dorsales y palmares; zona 3 , que sólo contiene la rama superficial o sensitiva que envía algunas ramas para el músculo palmaris brevis e inerva la piel sólo de la cara palmar de la mitad cubital del $4^{\circ}$ dedo y la del $5^{\circ}$ dedo. Además, el nervio digital propio de la mitad cubital del $\mathrm{V}$ dedo, se

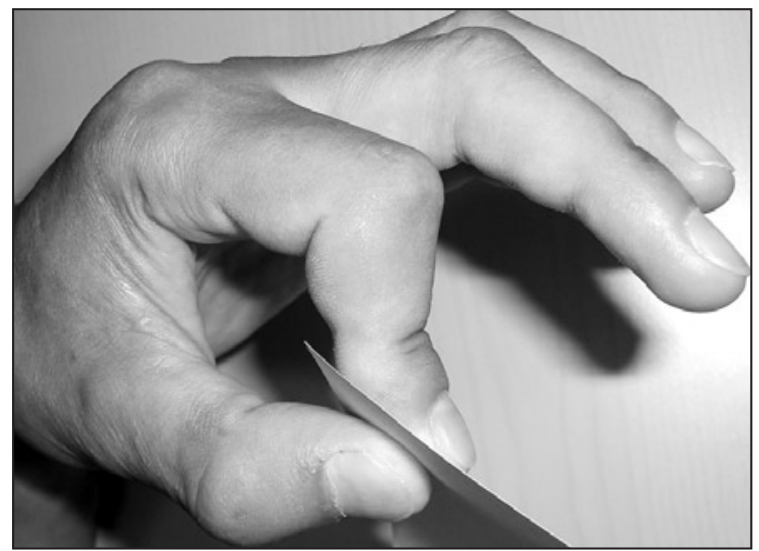

Figura 2: Caso 3. Forma tras fractura abierta de EDR, mano izquierda. Signo de Froment. caracteriza por diferenciarse directamente de la rama superficial ${ }^{2}$.

\section{MATERIAL Y MÉTODO}

Presentamos 6 casos de lesión del nervio cubital en el canal de Guyon, de diferentes etiologías, tratados entre los años 2004 y 2008 (Tabla I).

Se trataba de 5 hombres y 1 mujer, con edades comprendidas entre los 20 y 49 años (media de 32,5 años). La lesión se produjo en 3 casos en la mano derecha y en 3 en la izquierda, siendo la mano derecha la dominante en todos los casos. 3 lesiones ocurrieron en la zona 1 y 3 en la zona 2 del canal de Guyon.

En cuanto a la etiología, 3 casos tuvieron un origen traumático, tras accidente laboral, manifestados de forma aguda o subaguda: 2 formas de presentación aguda debidas a un traumatismo penetrante, uno con un cristal en zona 1 (mensajero, tras accidente de moto) (Caso 1) (Figura 1) y otro con un objeto punzante en zona 2 (agricultor) (Caso 2), que se intervinieron quirúrgicamente a la semana y a las 24 horas de la lesión respectivamente; la forma subaguda se presentó tras una fractura abierta grado I de la extremidad distal del radio (EDR) tratada incruentamente, haciéndose evidente la afectación nerviosa en zona 1 tras la retirada del yeso a las 6 semanas (trabajador de la construcción) (Caso 3) (Figura 2), único caso que se trato de forma conservadora.

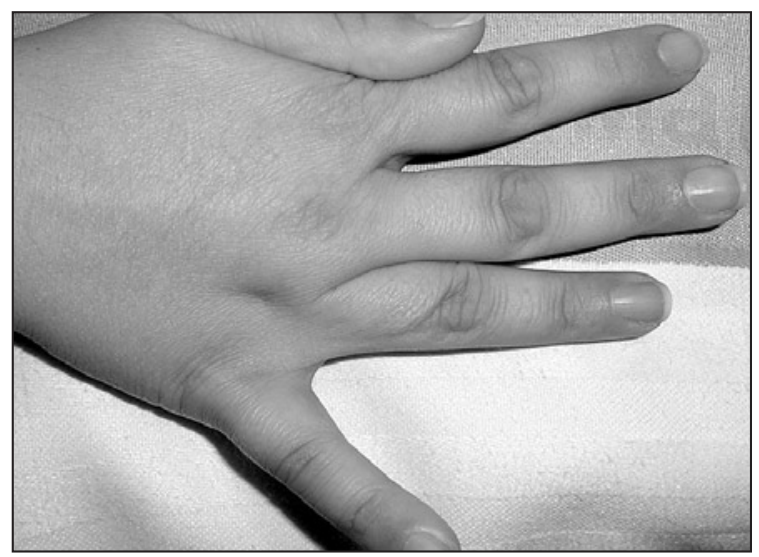

Figura 3: Caso 4. Forma iatrogénica. Signo de Wartenberg o de abducción mantenida del $\mathrm{V}$ dedo. 

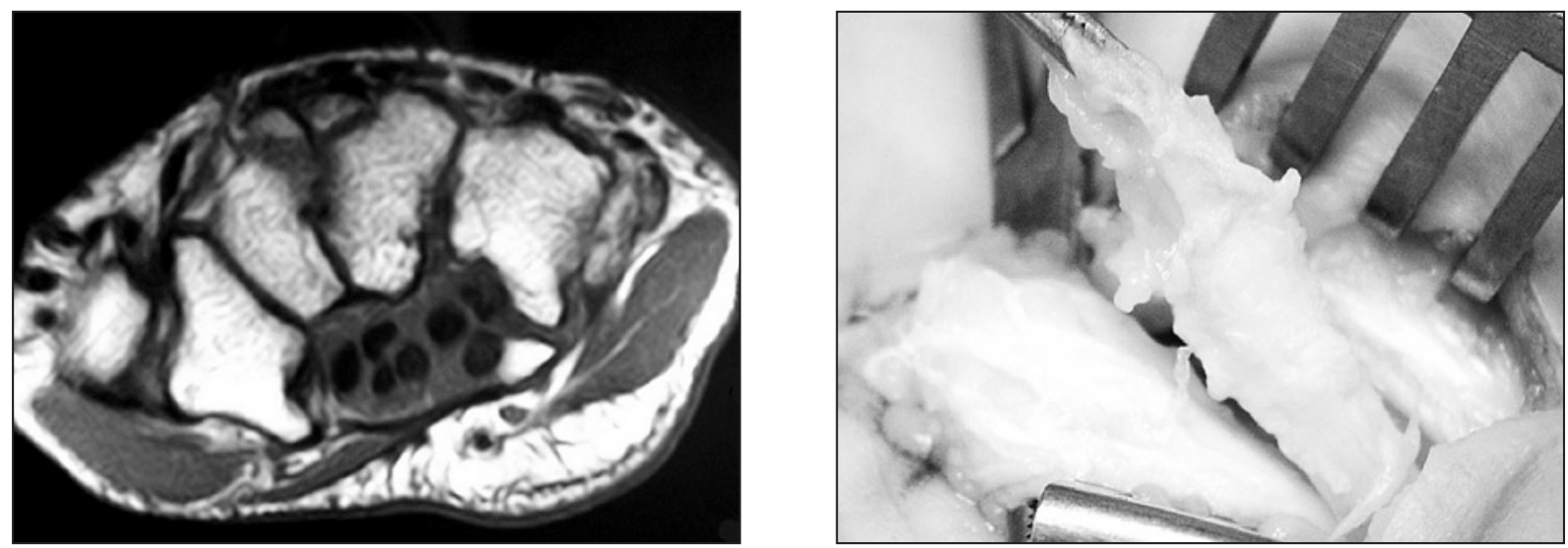

Figura 4: Caso 5. A: RM de fractura apófisis unciforme del ganchoso. B: Rotura degenerativa del flexor digitorum profundus del V dedo

Tres casos se presentaron de forma crónica, también por diferentes etiologías: una lesión iatrogénica en zona 1, tras la liberación del nervio mediano en el canal carpiano, realizada un año antes (panadera) (Caso 4) (Figura 3); una lesión en zona 2, tras una fractura de la apófisis unciforme del ganchoso, diagnosticada por RM, con rotura degenerativa del tendón flexor digitorum profundus del $\mathrm{V}$ dedo hallada en la cirugía (cocinero) (Caso 5) (Figura 4); y una lesión en zona 2 debida a una artrosis del carpo consecuencia de una seudoartrosis de escafoides, diagnosticada también por RM (tapicero) (Caso 6). En estos dos últimos casos, los pacientes apenas recordaban el traumatismo sufrido varios años antes y desconocían haber sufrido ningún tipo de fractura, acudiendo por presentar clínica dolorosa y disminución de fuerza con un EMG positivo, realizándose el diagnostico de la etiología secundariamente, tras el estudio por Rx y RM.

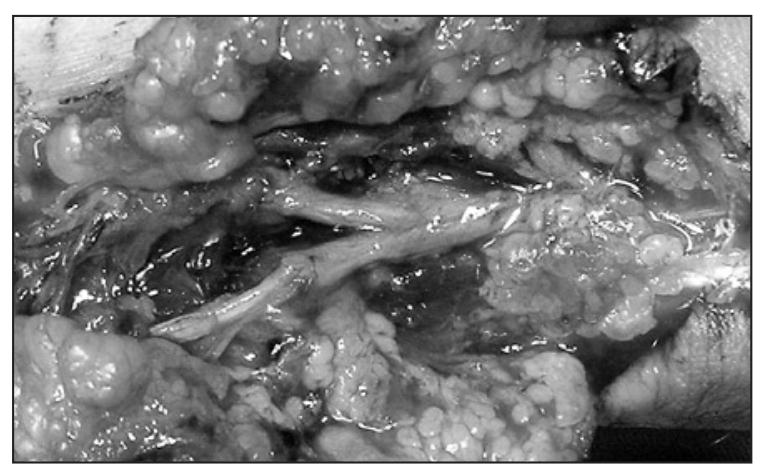

Figura 5: Caso 1. Sutura T-T de sección completa en zona 1 de las ramas sensitiva y motora.

\section{RESULTADO}

Sólo un caso se trató de forma conservadora (secundario a una fractura de EDR) (Caso 3) y el resto de forma quirúrgica. En las lesiones por un traumatismo penetrante, se realizo la apertura del canal de Guyon y la reparación microquirúrgica mediante sutura epiperineural término-terminal (T-T), en el paciente con lesión en zona 1 (Caso 1) (Figura 5), de una sección completa de la rama motora y de la rama sensitiva; y en el paciente con lesión en zona 2 (Caso 2), de una sección parcial de la rama motora. También en la forma iatrogénica con lesión en zona 1 (Caso 4), se realizo la resección del neuroma y sutura microquirúrgica T-T de las ramas terminales sensitiva y motora (Figura 6).

En las formas presentadas de forma crónica como secuela de fracturas, se realizó la apertura del canal de Guyon, con liberación de ambas ramas del nervio cubital (Casos 5 y 6). En el caso debido a la fractura de la apófisis unciforme del ganchoso (Caso 5), además se liberó el canal carpiano, y se hizo una sinovectomía de flexores

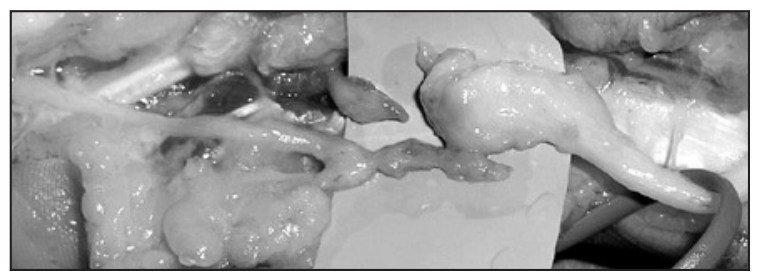

Figura 6: Caso 4. Neuromas de lesión iatrogénica en zona 1 de las ramas sensitiva y motora. 
y una tenodesis del flexor digitorum profundus del V dedo al IV dedo.

La apertura del canal de Guyon, se realizó a través de una incisión centrada en el lado cubital, en el eje de la eminencia hipotenar, por dentro del pisiforme, que se extiende desde el pliegue de flexión proximal de la muñeca al pliegue palmar proximal, y que se puede prolongar hacia la palma de la mano, para explorar la rama motriz. Se diseca el subcutáneo y se abre primero el hiato proximal o superficial de arriba abajo, seccionando longitudinalmente las fibras transversales del palmaris brevis, lo que permite hacer una primera exploración del nervio y liberar la rama superficial o sensitiva, para posteriormente abrir la arcada de los hipotenares, a nivel del hiato distal profundo, formado entre el abductor digiti minimi y el flexor digiti minimi brevis, para liberar la rama profunda o motora ${ }^{1}$ (Figura 7).

En todos los casos, tras la cirugía, se indicó tratamiento médico con pregabalina y tratamiento rehabilitador más ortesis activas de extensión para el IV-V dedo, mismo tratamiento que se indico para el único caso que se trato de forma conservadora, aparecido de forma secundaria a una fractura de la EDR.

El período de seguimiento fue variable, siendo dados de alta con reincorporación a su trabajo habitual, los pacientes con lesión traumática, al mes (lesión parcial de la rama motora) o los 3 meses (lesión completa y forma tratada conservadoramente). En todos los casos, se observo una desaparición de las parestesias y del dolor, sobre todo en las formas compresivas, con recuperación de fuerza del puño y de la pinza, y desaparición del signo de Froment, recuperación de la garra del IV-V dedo y recuperación de la abducción y aducción de los dedos, permaneciendo en todos los casos un ligero déficit de aducción del $\mathrm{V}$ dedo, que desaparece con el tiempo, no habiendo precisado ningún paciente de corrección quirúrgica. Curiosamente, se observó un déficit de aducción entre el III y IV dedo en la paciente con una lesión iatrogénica (Caso 4).

\section{DISCUSIÓN}

Las lesiones del nervio cubital en el canal de Guyon son raras, siendo la tercera en frecuen-

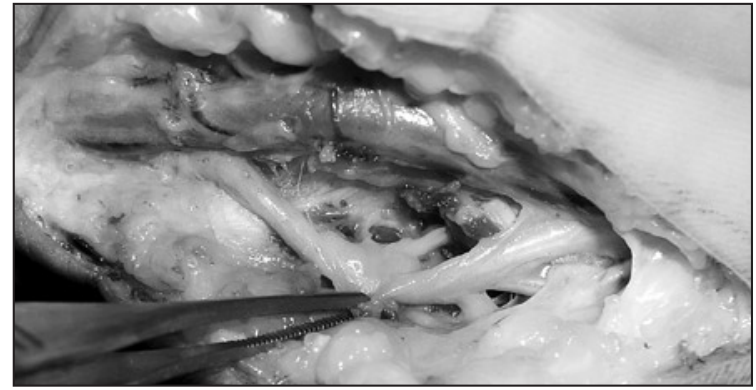

Figura 7: Caso 6 (Mano izquierda). Apertura del hiato superficial y profundo del canal de Guyon.

cia de los síndromes compresivos del miembro superior (después de la compresión del nervio mediano en el canal carpiano y del nervio cubital en el codo $)^{2}$, y, a diferencia de estos, es raro que aparezca de forma idiopática, siendo más frecuente que se manifieste de forma secundaria a causas locales: tumorales, variaciones anatómicas, traumáticas por fracturas o luxaciones de la muñeca y del carpo, degenerativas, por microtraumatismos profesionales (talla y pulido de vidrio, trabajo de zapatería, peleteros, herreros, etc) o deportivos por presión prolongada sobre el talón de la mano ${ }^{1,2}$, siendo una lesión que se ha observado con cierta frecuencia en los ciclis$\operatorname{tas}^{3,4}$. Dentro de las lesiones traumáticas, con lesión del nervio cubital y sus ramas, la lesión aislada de la rama motora tras un traumatismo penetrante es muy rara ${ }^{5}$.

El diagnóstico es fundamentalmente clínico y tras el estudio EMG, pero las pruebas de imagen ( $\mathrm{Rx}, \mathrm{TAC}$, Doppler, RM) pueden ayudar al diagnóstico, pues dan imágenes de la muñeca útiles para el estudio de la anatomía de la eminencia hipotenar, del canal de Guyon, de la arteria y nervio cubital, así como nos permiten diagnosticar, las posibles causas del dolor y de los síntomas, en las formas compresivas (fracturas, artrosis, tendinopatías, anomalías musculares, gangliones, quistes o tumores) ${ }^{6,7}$.

La principal diferencia clínica con la compresión del nervio cubital en el codo, está en que las alteraciones sensitivas sólo van a existir en la cara palmar de la mitad cubital de la mano, siendo el déficit motor característico de las formas evolucionadas, sin que exista afectación de la musculatura extrínseca (tendón del flexor carpi ulnaris y flexor digitorum profundus del $4^{\circ}$ y $5^{\circ}$ dedo), sino sólo 
parálisis de los músculos intrínsecos con déficit para la abducción y aducción de los dedos, más una atrofia hipotenar e intermetacarpiana o parrilla cubital, siendo más característico de lesión del nervio cubital, la atrofia del primer espacio interóseo dorsal, deformidad en garra del $4^{\circ}$ y $5^{\circ}$ dedo, debilidad de la pinza con signo de Froment positivo por la parálisis del adductor pollicis y el flexor pollicis brevis, y el signo de Wartenberg o de abducción mantenida del meñique porque el abductor digiti minimi permanece indemne ${ }^{1,2}$. Sin embargo, sí la causa de la lesión es traumática, entonces los síntomas motores y sensitivos, aparecen inmediatamente después de la lesión ${ }^{5}$.

El tratamiento es habitualmente quirúrgico ${ }^{1}$, orientado a liberar el nervio, mediante la apertura del canal de Guyon, y a la reparación de la lesión nerviosa, mediante la sutura microqui- rúrgica epiperineural T-T con o sin la necesidad de injerto nervioso ${ }^{5}$, o a eliminar la causa de la compresión.

Por otro lado, las relaciones anatómicas de este espacio, deben ser conocidas, para evitar complicaciones en la cirugía del canal carpiano, teniendo en cuenta que el borde radial del canal de Guyon no está constituido por el gancho o apófisis unciforme del ganchoso (a diferencia del canal carpiano, donde su pared interna está constituida por el pisiforme y el gancho del ganchoso), presentando el suelo del canal, constituido por el ligamento anular anterior del carpo (techo del canal carpiano), un segmento central, que contiene sólo tejido adiposo, a través del cuál se puede originar la lesión del nervio cubital en el canal de Guyon, al realizar la liberación del nervio mediano en el canal carpiano ${ }^{8}$.

\section{BIBLIOGRAFÍA}

1. Moutet F. Compression du nerf ulnaire á la loge de Guyon. En: Syndromes canalaires du membre supérieur. Chir Main, 2004 ; 23 (Supp 1): S134-40.

2. Allieu Y, Camas M Roux JL. Síndrome de los canales y de los desfiladeros (con excepción del canal carpiano). Encycl Méd Chir, Appareil locomoteur. Paris : Edit Elselvier, 1997: 14462 (18 p).

3. Akuthota V, Plastaras C, Lindberg $\mathrm{K}$, et al. The effect of longdistance bicycling on ulnar and median nerves: an electrophysiologic evaluation of cyclist palsy. Am J Sports Med, 2005; 33: 1224-30.

4. Capitani D, Beer S. Handlebar palsy-a compression syndrome of the deep terminal (motor) branch of the ulnar nerve in biking. J Neurol, 2002; 249: 1441-5.

5. Ozaksar K, Toros T, Kayalar M, et al. Isolated severance of the intrinsic motor branch of the ulnar nerve caused by stab injuries to the hand: A report of three cases. Acta Orthop Traumatol Turc, 2007; 41: 249-52.

6. Andreisek G, Crook DW, Burg $D$, et al. Peripheral neuropa- thies of the median, radial and ulnar nerves: MR imaging features. Radiographics, 2006; 26: 1267-87.

7. Blum AG, Zabel JP, Kohlmann $R$, et al. Pathologic conditions of the hipotenar eminence: evaluation with multidetector CT and MR imaging. Radiographics, 2006; 26: 1021-44.

8. Cobb TK, Carmichael SW, Cooney WP. Guyon's canal revisited: an anatomic study of the carpal ulnar neurovascular space. J Hand Surg Am, 1996; 21: 861-9. 